\title{
Clinical and bacteriological profile of diabetic foot infections in a tertiary care
}

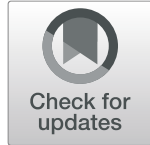

\author{
Teik Chiang Goh ${ }^{1,2}$, Mohd Yazid Bajuri ${ }^{{ }^{*}}$ (D), Sivapathasundaram C. Nadarajah², Abdul Halim Abdul Rashid ${ }^{1}$, \\ Suhaila Baharuddin ${ }^{3}$ and Kamarul Syariza Zamri
}

\begin{abstract}
Background: Diabetic foot infection is a worldwide health problem is commonly encountered in daily practice. This study was conducted to identify the microbiological profile and antibiotic sensitivity patterns of causative agents identified from diabetic foot infections (DFls). In addition, the assessment included probable risk factors contributing to infection of ulcers that harbour multidrug-resistant organisms (MDROs) and their outcomes.
\end{abstract}

Methods: We carried out a prospective analysis based on the DFI samples collected from 2016 till 2018. Specimens were cultured with optimal techniques in addition to antibiotic susceptibility based on recommendations from The Clinical and Laboratory Standards Institute (CLSI). A total of 1040 pathogens were isolated with an average of 1.9 pathogens per lesion in 550 patients who were identified with having DFls during this interval.

Results: A higher percentage of Gram-negative pathogens (54\%) were identified as compared with Gram-positive pathogens (33\%) or anaerobes (12\%). A total of $85 \%$ of the patients were found to have polymicrobial infections. Pseudomonas aeruginosa (19\%), Staphylococcus aureus (11\%) and Bacteroides species (8\%) appeared to be the predominant organisms isolated. In the management of Gram-positive bacteria, the most efficacious treatment was seen with the use of Vancomycin, while Imipenem and Amikacin proved to be effective in the treatment of Gramnegative bacteria.

Conclusion: DFI's are common among Malaysians with diabetes, with a majority of cases displaying polymicrobial aetiology with multi-drug resistant isolates. The data obtained from this study will be valuable in aiding future empirical treatment guidelines in the treatment of DFIs. This study investigated the microbiology of DFIs and their resistance to antibiotics in patients with DFIs that were managed at a Tertiary Care Centre in Malaysia.

Keywords: Bacteriology profile, Diabetic foot infections, Microbial sensitivity tests, Retrospective studies

\section{Background}

A worrying global health predicament in the modern age is Diabetes Mellitus (DM). Approximately 150-170 million of the world's population is suffering from this condition and its prevalence will most likely multiply by two folds by 2025 as per the World Health Organization (WHO) reports [1]. The WHO has identified the leading

\footnotetext{
* Correspondence: ezeds007@yahoo.com.my

'Department of Orthopaedic and Traumatology, Universiti Kebangsaan Malaysia Medical Centre, Hospital Canselor Tunku Muhriz, 56000 Cheras, Malaysia

Full list of author information is available at the end of the article
}

10 countries in Asia with a large number of people with DM including India, Japan, China, Korea, Bangladesh, Thailand, Indonesia, Philippines and Malaysia. Malaysia is forecast to have an estimated leap from 2.5 million people with DM in the year 2018 to a drastic and frightening increase to 5 million people by the year 2030 [2]. The preponderance of DM in Malaysia is almost 32\% of the population which has significantly increased from $0.6 \%$ in 1960 to $2.1 \%$ in $1982,6.3 \%$ in $1986,8.3 \%$ in 1996 and $14 \%$ in 1998 [3, 4]. DM is now estimated to affect the lives of $16-18 \%$ of Malaysians [3, 5]. Patients who do 
not have good control of their DM are at risk of developing complications arising from diabetes which are macrovascular and microvascular. Among these complications are neuropathy, retinopathy and pedal ulcers with or without gangrene [3]. Almost 15\% of patients with DM eventually end up developing foot ulcers that can progress to osteomyelitis [6]. The progression of these wounds from superficial to debilitating infections are facilitated by impaired defence mechanisms of the body and delayed treatment. The subcutaneous tissue infections have the ability to spread to the deeper structures which will ultimately progress into complications including gangrenous changes and also amputations [7]. Poor control of DM puts a patient at higher risk of skin infections as being in a state of hyperglycaemia impairs the efficiency of the body's immune or defence mechanism [8]. Diabetic foot infections (DFIs) are complex, rampant and costly ramifications of DM. These infections account for the majority of DM related hospitalisations other than non-traumatic amputations [9]. Few have studied the relationship between number of organisms identified and the types of infections that occur from it. In 2003, Frykberg concluded that most mild infections are commonly monomicrobial in which Gram-positive cocci such as Streptococcus species and Staphylococcus aureus were identified.

Contrarily, the more severe forms of DFI's are commonly polymicrobial in which the causative organism include Gram-negative bacilli (e.g., Klebsiella species., Proteus species., Escherichia coli, Pseudomonas species.) aerobic Gram-positive cocci, and anaerobes [10].

Prompt initiation of ideal therapy for DFIs plays a role in the reduction of morbidities interlinked with infections. Despite that, there can be a reduction in the number of hospitalisations and their durations in addition to reducing the incidence of major limb amputation. There are many factors that contribute to successful clinical outcomes, which include prompt lesion recognition followed by host factor modification, expeditious commencement of ideal antibiotic therapy plus efficient surgical intervention which includes debridement of bone and soft necrotic tissue. Prompt antibiotic therapy should be initiated to improve limb saving probabilities as many DFIs are true emergencies [11]. The aim of this study was to further enhance understanding on the bacteriology of diabetic foot ulcers alongside the assessment of the in vitro antimicrobial susceptibility of the offending pathogens in the General Hospital of Melaka, which is located in the historical state of Melaka, Malaysia and Universiti Kebangsaan Malaysia Medical Centre which is located in central Kuala Lumpur, both with a capacity to accommodate nearly one thousand patients. These two hospitals are major general tertiary care centres in their respective states and are the primary centres that receive referrals for further management of DFI's.

\section{Materials and methods}

This prospective study included 550 patients with longstanding and newly diagnosed DM who presented with lower extremity infections requiring admission to the General Hospital of Melaka and Universiti Kebangsaan Malaysia Medical Centre. This study was conducted over a period of 2 years between 1st February 2016 and 1st February 2018. There was no duplications in the recruitment of patients in the study. Patients underwent clinical assessment and proper grading of their foot lesions was carried out in accordance with the DFI severity classification systems including the Wagner Classification System and the University of Texas Wound Classification System [12]. The classifications of wounds were according to the severity which was mild, moderate or severe based on the size, depth and level of the tissue involved in the infection, presence of metabolic instability or any systemic manifestations of infection. Briefings were given to patients regarding the details of the study. Patient information including age, gender, type of diabetes and duration of DFI's were recorded. Various specimens (wound exudates, pus or tissue biopsy) from the ulcerated regions were collected for microbiological studies. The surface of those regions was thoroughly rinsed with sterile normal saline solution and pus samples were taken using sterile cotton swabs. A total of 550 patients were identified from request forms and their clinical specimens were subsequently dispatched to the microbiology laboratory for further analysis. The forms were designed to provide pertinent demographic data and clinical information including a patient's age, gender, race, nature of the specimen, examination required, diagnosis and details of antimicrobial therapy. The request forms were completed and signed by the respective treating medical officers. Data were recorded according to patient's age, gender, ethnicity alongside nature of the clinical specimen, species of the isolated pathogen and antibiogram of the clinical isolates.

\section{Wagner's classification of diabetic foot ulcers}

Grade 0: no ulcer in a high-risk foot.

Grade 1: superficial ulcer involving the full skin thickness but not underlying tissues.

Grade 2: deep ulcer, penetrating down to ligaments and muscles, but no bone involvement or abscess formation.

Grade 3: deep ulcer with cellulitis or abscess formation, often with osteomyelitis.

Grade 4: localized gangrene.

Grade 5: extensive gangrene involving the whole foot. 


\section{University of Texas Wound Classification System of diabetic foot ulcers}

Grade IA: not infected, no ischemic superficial ulceration.

Grade IB: infected, no ischemic superficial ulceration.

Grade IC: ischemic, no infected superficial ulceration.

Grade ID: ischemic, infected superficial ulceration.

Grade IIA: not infected, no ischemic ulcer that penetrates to capsule or bone.

Grade IIB: infected, no ischemic ulcer that penetrates to capsule or bone.

Grade IIC: ischemic, no infected ulcer that penetrates to capsule or bone.

Laboratory investigations and hospital records of patients recruited into the study between February 2016 to February 2018 were reviewed meticulously. The technique of specimen collection included clinically infected wounds while excluding superficial or colonizing organisms. Specimen isolates were not duplicated in this study. The specimen cultures were obtained once the wound surface had been irrigated with copious normal saline, and we subsequently debrided the superficial tissue and separated them from the exudates to isolate the colonizing pathogenic flora. Specimens were obtained by curetting the ulcer base post debridement and by aspirating the infected skin or deep tissues. The specimens underwent gram staining and inoculation on both selective and non-selective media including MacConkey agar (Oxoid), blood agar (BA; Oxoid, Basingstoke, UK), chocolate agar and $5 \%(\mathrm{v} / \mathrm{v})$ BA supplemented with vitamin $\mathrm{K} 1(1 \mu \mathrm{g} / \mathrm{ml})$, gentamicin $(75 \mu \mathrm{g} / \mathrm{ml})(\mathrm{GBA})$ and haemin $(5 \mu \mathrm{g} / \mathrm{ml})$. The inoculated plates were incubated under conducive atmospheric conditions for 24-48 h to enable bacterial isolation. Isolated organisms were identified by traditional microbiological methods: Analytical profile index 20 (API 20) Strep for streptococci and enterococci, API 20A (bioMérieux, Marcy l'Etoile, France) and GLC (Chromopak, CP9001, The Netherlands) for anaerobes, API 20E for Gram-negative aerobes and API Staph for staphylococci. According to the standardised procedures described by Sharma, the bacteria were isolated and classified by microscopy, morphology and biochemical testing [13].

\section{Antimicrobial susceptibility testing}

In accordance with the guidelines of the Clinical and Laboratory Standards Institute (CLSI), confirmation of antimicrobial susceptibility of the bacterial isolates was carried out by using the disk diffusion method [14]. Isolated bacterial colonies were suspended in sterile distilled water and matched to the $0.5 \mathrm{McF}$ arland standard $[5,15]$. A sterile swab was soaked into the inoculums and subsequently used to streak the Mueller-Hinton agar plates for Gram-negative bacilli, Staphylococci, and
Enterococci. For Streptococci, the Mueller-Hinton agar was supplemented with $5 \%$ horse blood as described in the CLSI guidelines and followed by application of antibiotic discs on the plates. The inoculated agar plates were incubated at $35-37^{\circ} \mathrm{C}$ for $18-20 \mathrm{~h}$. Then, the diameter of the inhibition zone was interpreted based on the guideline suggested by the CLSI. Staphylococcus aureus was tested for Methicillin resistance using a $1 \mu \mathrm{g}$ Oxacillin disc. Reference strains of Escherichia coli and Pseudomonas aeruginosa were used as a control for Gramnegative bacteria and were included in the tests. Staphylococcus aureus and Enterococcus faecalis were used as Gram-positive control strains [14].

\section{Results}

\section{Demographic characteristics}

All 550 patients successfully recruited into the study were recruited. All had been diagnosed with Diabetic Foot Ulcer to participate in this particular study. The data of these patients were compiled and summarized in Table 1. In this study, 337 out of 550 (61\%) were male and 213 out of 550 (39\%) were female with a male-tofemale ratio of 1.58:1. The patients' age ranged from 37 to 91 years old with a mean of 56.7 years. Out of the total population, only $10(1.8 \%)$ patients presented with type $1 \mathrm{DM}$ and the rest $540(98.2 \%)$ were type $2 \mathrm{DM}$. The majority of patients were Malaysian nationals 544 (98.9\%). Indians and Malays accounted for $40 \%(n=220)$ and $39 \%(n=214)$ respectively, while the Chinese population accounted for $20 \%(n=110)$ followed by $1 \%(n=$ 6) of the others.

\section{Microbiology}

From 550 patients, we were able to isolate 1040 pathogens averaging at 1.8 per lesion. We included the specimens with clinically significant pathogens which were wound tissue $(220 ; 40 \%)$, swabs $(220 ; 40 \%)$, pus (83; $15 \%)$ and bone (27; 5\%). Patients were graded based on foot lesions and the number of bacterial isolates. Their

Table 1 Demographic distribution of 550 patients with diabetic foot infections

\begin{tabular}{|c|c|c|c|c|c|}
\hline \multirow{2}{*}{$\begin{array}{l}\text { Age } \\
\text { (years) }\end{array}$} & \multicolumn{2}{|c|}{ Malaysian $(\boldsymbol{n}=544)$} & \multicolumn{2}{|c|}{ Non-Malaysian $(\boldsymbol{n}=6)$} & \multirow{2}{*}{$\begin{array}{l}\text { Total no. (\%) } \\
(\boldsymbol{n}=550)\end{array}$} \\
\hline & $\bar{M}$ & $\mathrm{~F}$ & $\bar{M}$ & $\mathrm{~F}$ & \\
\hline $40 \leq$ & 27 & 26 & 0 & 1 & $54(9.8)$ \\
\hline $41-50$ & 65 & 46 & 3 & 2 & $116(21)$ \\
\hline $51-60$ & 137 & 55 & 0 & 0 & $192(35)$ \\
\hline $61-70$ & 85 & 67 & 0 & 0 & $152(27.6)$ \\
\hline $71-80$ & 18 & 15 & 0 & 0 & $33(6)$ \\
\hline $81-90$ & 2 & 0 & 0 & 0 & $2(0.4)$ \\
\hline$\geq 91$ & 0 & 1 & 0 & 0 & $1(0.2)$ \\
\hline Total & 334 & 210 & 3 & 3 & $550(100)$ \\
\hline
\end{tabular}


numbers are shown in Table 2. Table 3 presents the multiple organisms isolated from the DFIs. $54 \%$ of aerobic gram-negative bacteria were isolated and it was the most commonly isolated pathogens. The four most common microbial isolates were Pseudomonas aeruginosa (19\%), Staphylococcus aureus (11\%), Methicillin-resistant Staphylococcus aureus (8\%) and Bacteroides species (spp.) (8\%).

\section{Antibiotic susceptibility}

Analysis of this study showed that 468 (85\%) of the 550 infected patients had multiple microorganism infections. The most common microbial isolates in Gram-positive bacteria were Staphylococcus aureus (11\%), Gramnegative bacteria was Pseudomonas aeruginosa (19\%) and Anaerobes was Bacteroides species (8\%) respectively.

Table 4 illustrates the antimicrobial susceptibility pattern of the Gram-positive cocci. Out of the 150 Staphylococcus aureus isolates, 94.8, 62.0, and 56\% were penicillin-resistant, tetracycline and erythromycin, respectively. Vancomycin, the most sensitive antibiotic for Staphylococcus aureus organism. The Enterococcus spp. were susceptible to vancomycin and tetracycline, however, 63.5 and $15.9 \%$ were resistant to erythromycin and penicillin, respectively. The Group B Streptococcus isolates were susceptible to co-amoxiclav, penicillin, and ampicillin, but $51 \%$ were resistant to tetracycline. Table 5 demonstrates the pattern of antibiotic resistance for Gram-negative bacilli. Imipenem and Amikacin were the most active antimicrobial agents tested against all of the microbial isolates. Pseudomonas aeruginosa resistant towards Ciprofloxacin and Piperacillin-Tazobactam, both 18\% resistance. As for Proteus species and Klebsiella pneumonia, both were highly resistant towards Ampicillin (45\% \& 79\% respectively). The antibiotic susceptibility pattern of isolated anaerobes is shown in Table 6 . Amoxicillin, Ampicillin, Cefoxitin, Metronidazole and Piperacillin/Tazobactam showed excellent sensitivity against all anaerobes; there were no resistance towards these antibiotics. Nonetheless, $67 \%$ of the anaerobic

Table 2 The number of patients in each grade of diabetic foot infection and the total number of bacteria isolated from different grades of diabetic foot infection

\begin{tabular}{lllll}
\hline $\begin{array}{l}\text { Grades of } \\
\text { diabetic } \\
\text { foot }\end{array}$ & $\begin{array}{l}\text { No. of } \\
\text { patients }\end{array}$ & \multicolumn{2}{l}{ A positive culture for bacteria } & \\
\cline { 3 - 5 } infection & & & 2 & $>2$ \\
\hline Mild & 203 & 30 & 51 & 122 \\
Moderate & 227 & 34 & 57 & 136 \\
Severe & 120 & 18 & 30 & 72 \\
Total & 550 & 82 & 138 & 330 \\
& & $(15 \%)$ & $(25 \%)$ & $(60 \%)$ \\
\hline
\end{tabular}

Table 3 The proportion and frequency of microbial isolation from the samples

\begin{tabular}{|c|c|c|c|}
\hline \multirow[t]{2}{*}{ Bacteria } & Total number & Proportion & Frequency \\
\hline & \multicolumn{3}{|l|}{$\boldsymbol{N}=1040$} \\
\hline Gram-positive bacteria & 348 & 33 & 63 \\
\hline Staphylococcus aureus & 116 & 11 & 21 \\
\hline Methicillin-resistant S. aureus & 86 & 8 & 15 \\
\hline Enterococcus spp. & 63 & 6 & 11 \\
\hline Group B streptococci & 47 & 4 & 9 \\
\hline Other streptococci & 27 & 3 & 5 \\
\hline Other Staphylococcus spp. & 9 & 1 & 2 \\
\hline Gram-negative bacteria & 558 & 54 & 100 \\
\hline Pseudomonas aeruginosa & 192 & 19 & 35 \\
\hline Proteus mirabilis & 74 & 7 & 13 \\
\hline Klebsiella pneumonia & 71 & 7 & 13 \\
\hline Enterobacter spp. & 69 & 6 & 12 \\
\hline Escherichia coli & 60 & 6 & 10 \\
\hline Non-fermenters & 29 & 3 & 5 \\
\hline Morgenella spp. & 20 & 2 & 4 \\
\hline Other Gram-negative & 16 & 2 & 3 \\
\hline Serratia marcescens & 15 & 1 & 3 \\
\hline Citrobacter spp. & 12 & 1 & 2 \\
\hline Anaerobes & 123 & 12 & 22 \\
\hline Bacteroides spp. & 84 & 8 & 15 \\
\hline Anaerobic Streptococci & 18 & 2 & 3 \\
\hline Microaerop Streptococci & 10 & 1 & 2 \\
\hline Candida spp. & 9 & 1 & 2 \\
\hline Clostridium spp. & 6 & 0.5 & 1 \\
\hline Fusobacterium spp. & 5 & 0.5 & 1 \\
\hline
\end{tabular}

Proportion $=$ the percentage of isolates organism divided into the total number of isolated organisms.

Frequency $=$ the percentage of the organisms divided into the total number of patients.

streptococci and $32 \%$ of the Bacteroides species were resistant towards clindamycin.

\section{Discussion}

DFIs are a burgeoning predicament in Malaysia owing to the high incidence of DM among Malaysians where 1/ 5 th of the population aged more than 30 years old were diagnosed with DM Type 2 reported by Bajuri et al. [16]. Our particular study features significant elements including the etiological agents of DFIs which are predominantly polymicrobial and contain both Gram-positive and Gram-negative bacteria. Anaerobes contribute to the infection by approximately 0.25 anaerobic bacteria per culture-positive specimen, with the majority of the aetiological agents being multi-resistant. When detected in DFI's, anaerobes are commonly isolated from cultures that concurrently harbour aerobes. Notably, the isolation 
Table 4 Resistance pattern for most common Gram-positive cocci towards antimicrobials

\begin{tabular}{|c|c|c|c|c|c|c|}
\hline \multirow[t]{3}{*}{ Antimicrobial agents } & \multicolumn{6}{|c|}{ Gram-positive organisms (\% resistant) } \\
\hline & \multicolumn{2}{|c|}{ S. aureus } & \multicolumn{2}{|c|}{ Enterococcus spp. } & \multicolumn{2}{|c|}{ Group B Streptococcus } \\
\hline & $\boldsymbol{n}=116$ & (\%) & $n=63$ & (\%) & $\boldsymbol{n}=47$ & $(\%)$ \\
\hline Ampicillin & 59 & 50.8 & - & - & 0 & 0.0 \\
\hline Clindamycin & 56 & 48.2 & - & - & 5 & 10.6 \\
\hline Cloxacillin & 60 & 51.7 & - & - & - & - \\
\hline Co-amoxiclav & 59 & 50.8 & - & - & 0 & 0.0 \\
\hline Erythromycin & 65 & 56.0 & 40 & 63.5 & 8 & 17.0 \\
\hline Fusidic acid & 60 & 51.7 & - & - & - & - \\
\hline Gentamicin & 52 & 44.8 & 1 & 1.6 & 2 & 4.3 \\
\hline Penicillin & 110 & 94.8 & 10 & 15.9 & 0 & 0.0 \\
\hline Rifampicin & 1 & 0.8 & - & - & - & - \\
\hline Sulfamethoxazole-Trimethoprim & 30 & 25.8 & - & - & - & - \\
\hline Tetracycline & 72 & 62.0 & 0 & 0.0 & 24 & 51.0 \\
\hline Vancomycin & 0 & 0.0 & 0 & 0.0 & 0 & 0.0 \\
\hline
\end{tabular}

rate of anaerobes was lower than expected in our study despite its prevalence, especially in comparison with a report by Goldstein et al. [17]. There are many factors that may have contributed to this outcome which include suboptimal sampling or transportation of samples in addition to the use of inappropriate mediums. Previous studies stated that DFI's predominantly and usually harbour Gram-positive aerobes [18]. On the contrary, most of our patient's, the major infective organisms were gram-negative bacteria [19].

We discovered that the most common single pathogen isolation in diabetic foot infections was S. Aureus [11, 20, 21]. The prevalence of S. Aureus was 76 and $78 \%$ in studies according to Goldstein et al. and Kajetan et al.
[21-23]. However, we have reported a much lower prevalence when compared with earlier reports. This contrast is to be expected as the majority of our patients had severe forms of DFIs which are commonly polymicrobial when compared with mild DFIs that are usually associated with aerobic Gram-positive cocci, such as S. Aureus. Unfortunately, for the past two decades, MRSA has been a pathogen of concern especially with the evolution of community-acquired MRSA [13]. Our study concluded that the MRSA isolation rate increased to nearly four-folds when compared with previous Malaysian studies [5]. This development is likely due to a lack of strict antibiotic prescription guidelines alongside the lack of adherence to infection control measures in the

Table 5 Resistance pattern of most common Gram-negative organisms towards antimicrobials

\begin{tabular}{|c|c|c|c|c|c|c|}
\hline \multirow[t]{3}{*}{ Antimicrobial agents } & \multicolumn{6}{|c|}{ Gram-negative organisms (\% resistant) } \\
\hline & \multicolumn{2}{|c|}{ P. aeruginosa } & \multicolumn{2}{|c|}{ Proteus spp. } & \multicolumn{2}{|c|}{ K. pneumonia } \\
\hline & $\boldsymbol{n}=192$ & $(\%)$ & $\boldsymbol{n}=74$ & $(\%)$ & $\boldsymbol{n}=71$ & (\%) \\
\hline Amikacin & 15 & 8 & 0 & 0 & 0 & 0 \\
\hline Amoxicillin clavulanic acid & 22 & 11 & 17 & 23 & 13 & 18 \\
\hline Ampicillin & - & - & 33 & 45 & 56 & 79 \\
\hline Cefotaxime & - & - & 0 & 0 & 5 & 7 \\
\hline Ceftazidime & 20 & 10 & - & - & - & - \\
\hline Cefuroxime & 2 & 1 & 6 & 8 & 6 & 8 \\
\hline Ciprofloxacin & 34 & 18 & 12 & 16 & 10 & 14 \\
\hline Gentamicin & 16 & 8 & 13 & 18 & 8 & 11 \\
\hline Imipenem & 17 & 9 & 0 & 0 & 0 & 0 \\
\hline Piperacillin & 17 & 9 & 10 & 14 & 27 & 38 \\
\hline Piperacillin-Tazobactam & 34 & 18 & 0 & 0 & 3 & 4 \\
\hline Sulphonamides Trimethoprim & - & - & 17 & 23 & - & - \\
\hline
\end{tabular}


Table 6 Susceptibility pattern of most common anaerobic bacterial isolates towards antimicrobials

\begin{tabular}{|c|c|c|c|c|c|c|c|c|}
\hline \multirow[t]{3}{*}{ Antimicrobial agents } & \multicolumn{8}{|c|}{ Proportion susceptible (\%) } \\
\hline & \multicolumn{2}{|c|}{ Anaerobic Streptococci } & \multicolumn{2}{|c|}{ Bacteroides species } & \multicolumn{2}{|c|}{ Clostridium species } & \multicolumn{2}{|c|}{ Fusobacterium specie } \\
\hline & $\boldsymbol{n}=18$ & $(\%)$ & $\boldsymbol{n}=84$ & $(\%)$ & $\boldsymbol{n}=6$ & $(\%)$ & $\boldsymbol{n}=5$ & $(\%)$ \\
\hline Amoxicillin & - & - & - & - & - & - & - & - \\
\hline Ampicillin/CA & 18 & 100 & 84 & 100 & 6 & 100 & 5 & 100 \\
\hline Erythromycin & 18 & 100 & 25 & 30 & - & - & - & - \\
\hline Cefoxitin & 18 & 100 & 84 & 100 & 6 & 100 & - & - \\
\hline Clindamycin & 12 & 67 & 27 & 32 & - & - & - & - \\
\hline Metronidazole & 18 & 100 & 84 & 100 & 6 & 100 & 5 & 100 \\
\hline Penicillin-G & 18 & 100 & 3 & 4 & 6 & 100 & - & - \\
\hline Piperacillin & 18 & 100 & 75 & 89 & 6 & 100 & - & - \\
\hline Piperacillin/tazobactam & 18 & 100 & 84 & 100 & 6 & 100 & 5 & 100 \\
\hline Tetracycline & - & - & - & - & - & - & - & - \\
\hline Vancomycin & - & - & - & - & - & - & - & - \\
\hline
\end{tabular}

hospital with a higher prevalence of MRSA in the community. If not rectified, these practices will likely alter empirical antimicrobial therapy.

In addition, approximately $25 \%$ of our patients demonstrated Streptococci cultures with a large number being S. agalactiae which is also known as group B Streptococcus [24]. This finding came as no surprise as $S$. agalactiae is a commonly identified DFI pathogen [17, 18]. Enterococci are considered to be commensals and exhibit low virulence, excluding those who are in an immunocompromised state such as those with DM in which commensals may also become opportunistic pathogens. Despite that, based on previous reports by various investigators, a large number of our patients' DFIs were caused by other different organisms [17].

Previous antimicrobial use, prolonged hospitalizations, chronic wounds, and surgical interventions which are all distinctive of patients with this type of infection in our hospital are likely contributors to the comparatively large prevalence of P. Aeruginosa in this study. Moreover, a high proportion of E.Coli and Klebsiella spp. isolates were positive for ESBLs and comparison with studies in other regions deduced that the incidence of ESBL-producing Klebsiella spp. and E. coli was higher in our population of patients with DFIs [5]. Hence, routine screening towards ESBL-producing Enterobacteriaceae should be emphasised.

Piperacillin-Tazobactam, Amikacin and Imipenem were the greatest advantageous antimicrobials against aerobic Gram-negative bacteria. As for aerobic Grampositive cocci and anaerobes, the most effective antimicrobials where Vancomycin and Metronidazole respectively [25]. It is vital to ensure meticulous and thoughtful choices of antimicrobial therapy guided by culture findings in addition to the antimicrobial sensitivity patterns of the specific isolates. Our research has brought us to the conclusion that Ciprofloxacin is not to be recommended for empirical treatment in DFIs as it was particularly inactive against many strains of pathogens identified in the infections. Furthermore, initiation of treatment with broad-spectrum antimicrobials including Carbapenems and Piperacillin-Tazobactam for moreextensive chronic moderate and severe infections appears to be a safe measure. Definitive treatment can subsequently be initiated upon confirmation of offending pathogen via culture and sensitivity testing, susceptibility data and the patient's response to empirical therapy from a clinical aspect. In order to implement targeted and correct antimicrobial therapy, it is essential to have knowledge and awareness of the common offending pathogens in DFIs.

The management of DFIs often calls for combination therapy with surgical intervention in the form of drainage and debridement or osseous resection especially if osteomyelitis was present [26]. In recent studies, recommendations have included treatment with Hyperbaric Oxygen Therapy (HBOT) as an adjunctive treatment in managing chronic DFIs as they can accelerate wound healing and improve patients' quality of life [27]. The preference of antibiotic therapy is guided by the sensitivity of the bacterial pathogens encountered. Empirical antimicrobial therapy should utilise versatile antibiotics that have a wide coverage including the coverage of Gram-negative microorganisms, Gram-positive microorganisms and anaerobes if they are particularly suspected in infected foot ulcers. Detailed and meticulous microbiological workup is imperative to the selection of proper antibiotics for DFIs. Numerous drugs have been used to treat non-limb-threatening infections including Aminoglycosides, Ampicillin, Penicillin, Beta-lactamase 
inhibitors, third-generation Cephalosporins, Quinolones, Piperacillin-Tazobactam and Linezolid [13]. It was also found that Enterococci and anaerobes were not responsive to third-generation Cephalosporins, while Streptococci and anaerobes were said to have been less responsive to Fluoroquinolones [5, 28]. The limitation for this study is that the validated diabetic foot infection tool such as Infectious Diseases Society of America/ International Working Group (IDSA/IWGDF) classification was not used as it is not been practiced routinely in our centre. Despite that, there is no difference in the management of our diabetic foot infections in compare to the practice that has been published elsewhere.

\section{Conclusion}

The common aetiology of the DFIs in our patients was polymicrobial in nature in which P. Aeruginosa and S. Aureus were identified to be the most common agents and there was a low proportion of anaerobic organisms. Vancomycin, Piperacillin-Tazobactam or Imipenem appear to be suitable agents for empirical therapy according to antimicrobial susceptibility data. Clinicians are also encouraged to obtain adequate specimens after wound debridement for proper culture in addition to requesting for prompt microbiological reporting of all organisms which are obtained from the specimens. Considering the limited suitability of antibiotics, determination of the appropriate antimicrobial treatment should be guided by clinical correlation in addition to the local pattern of bacterial aetiology and its sensitivity. On the grounds that DFI's are of polymicrobial aetiology, it is unlikely for a single antimicrobial agent to be effective against all the probable organisms isolated from diabetic foot infections. The findings of this study further emphasise the need to select antimicrobial treatment should be guided by proven culture results and antimicrobial sensitivity patterns exhibited by isolates. Choosing empirical antibiotic therapy will be guided by many factors including overall patient condition, glycaemic control, the severity of infection, allergies towards medication, prior antibiotic usage, antibiotic activity, excretion and toxicity. It is imperative to identify causative agents, appropriate antibiotic therapy and management of complications of diabetic foot infections in order to accomplish optimal results.

\footnotetext{
Abbreviations

DFIs: Diabetic foot infections; MDROs: Multidrug-resistant organisms; CLSI: Clinical and Laboratory Standards Institute; DM: Diabetes Mellitus; WHO: World Health Organization; ESBLs: Extended spectrum beta-lactamases; MRSA: Methicillin-resistant Staphylococcus aureus; HBOT: Hyperbaric Oxygen Therapy; BA: Blood agar; API: Analytical profile index; SPP: Species; IDSA: Infectious Diseases Society of America; IWGDF: International Working Group of Diabetic Foot
}

\section{Authors' contributions}

TCG, MYB, SCN and AH contributed to conception and design of the study, carried out data collection, draft the manuscript and review it equally. SB interpret all the specimens and provided results from patients. KSZ coordinated and analysed and interpreted the data. All authors were involved in drafting the manuscript and have given final approval of the version to be published.

\section{Funding}

No funding received for this research.

\section{Availability of data and materials}

All data generated or analysed during this study are included in this published article.

Ethics approval and consent to participate

This study was reviewed and approved by the Ethics Committee of the National Medical Research Register (NMRR) using the following code NMRR-181093-41211.

\section{Consent for publication}

Patients were not required to give informed consent in this study as the analysis used anonymous clinical data that were obtained once patients agreed to treatment with written consent.

\section{Competing interests}

The authors declared that they have no competing interests.

\section{Author details}

'Department of Orthopaedic and Traumatology, Universiti Kebangsaan Malaysia Medical Centre, Hospital Canselor Tunku Muhriz, 56000 Cheras, Malaysia. ${ }^{2}$ Department of Orthopaedic and Traumatology, Hospital Melaka, 75400 Melaka, Malaysia. ${ }^{3}$ Department of Microbiology, Hospital Melaka, 75400 Melaka, Malaysia.

Received: 15 April 2020 Accepted: 11 June 2020

Published online: 16 June 2020

\section{References}

1. Moussa M, Alsaeid M, Abdella N, Refai T, Al-Sheikh N, Gomez J. Prevalence of type 2 diabetes mellitus among Kuwaiti children and adolescents. Med Princ Pract. 2008;17(4):270-5.

2. Zimmet P, Alberti K, Shaw J. Global and societal implications of the diabetes epidemic. Nature. 2001:414(6865):782-7.

3. Zaini A. Where is Malaysia in the midst of the Asian epidemic of diabetes mellitus? Diabetes Res Clin Pract. 2000;50:523-8.

4. Mustaffa BE. Diabetes epidemic in Malaysia. Med J Malays. 2004;59(3):295-6.

5. Raja NS. Microbiology of diabetic foot infections in a teaching hospital in Malaysia: a retrospective study of 194 cases. J Microbiol Immunol Infect. 2007:40:39-44.

6. Ramsey SD, Newton K, Blough D, McCulloch DK, Sandhu N, Reiber GE, Wagner EH. Incidence, outcomes, and cost of foot ulcers in patients with diabetes. Diabetes Care. 1999;22(3):382-7.

7. Al-Hamead Hefni A, Ibrahim A-MR, Attia KM, Moawad MM, El-ramah AF, Shahin MM, Al-Molla M, Abd A-SL. Bacteriological study of diabetic foot infection in Egypt. Arab Soc Med Res. 2013;8:26-32.

8. Jeffcoate W, Harding K. Diabetic foot ulcers. Lancet. 2003;361(9368):1545-51.

9. Lipsky B. A report from the international consensus on diagnosing and treating the infected diabetic foot. Diabetes Metab Res Rev. 2004;20(S1): S68-77.

10. Frykberg R. An evidence-based approach to diabetic foot infections. Am J Surg. 2003;186(5):44-54.

11. Lipsky BA, Berendt AR, Deery HG, Embil JM, Joseph WS, Karchmer AW, LeFrock JL, Lew DP, Mader JT, Norden C, Tan JS. Diagnosis and Treatment of Diabetic Foot Infections. Plast Reconstr Surg. 2006;117(SUPPLEMENT): 212S-38S.

12. Oyibo SO, Jude EB, Tarawneh I, Nguyen HC, Harkless LB, Boulton AJ. A comparison of two diabetic foot ulcer classification systems: the Wagner and the University of Texas wound classification systems. Diabetes Care. 2001;24(1):84-8

13. El-Tahawy AT. Bacteriology of diabetic foot. Saudi Med J. 2000;21(4):344-7. 
14. Wayne PA. Performance standards for antimicrobial disk susceptibility tests. Approved standard, Ninth edition. Document M2-A9. CLSI; 2006.

15. Wayne PA, National Committee for Clinical Laboratory Standards. Performance Standards for antimicrobial susceptibility testing: 14th Informational Supplement NCCLS Document M100-S14. 2004.

16. Padzil AWM, Bajuri MY. Virtual amputation as a conservative surgical approach in treating diabetic foot osteomyelitis- a case series. J Clin Diagn Res. 2019;13(3):1-4. https://doi.org/10.7860/JCDR/2019/39994.12648.

17. Citron DM, Goldstein EJC, Merriam CV, Lipsky BA, Abramson MA. Bacteriology of moderate-to-severe diabetic foot infections and in vitro activity of antimicrobial agents. J Clin Microbiol. 2007;45(9):2819-28.

18. Mantey I, Hill RL, Foster AV, Wilson S, Wade JJ, Edmonds ME. Infection with foot ulcers with Staphylococcus aureus associated with increase mortality in diabetic patients. Commun Dis Public Health. 2000;3(4):288-90.

19. Cauton-Valera R, Lianes LRI, Pena AC. Clinical, microbiological profile and outcome of diabetic patients with foot ulcers admitted at the Quirino memorial medical center: January 2000-may 2001. Philipp J Microbiol Infect Dis. 2001;30(3):101-7.

20. Abdulrazak A, Ibrahim Bitar ZI, Al-Shamali AA, Mobasher LA. Bacteriological study of diabetic foot infections. J Diabetes Complicat. 2005;19(3):138-41.

21. Goldstein $E$, Citron D, Nesbit C. Diabetic foot infections: bacteriology and activity of 10 oral antimicrobial agents against bacteria isolated from consecutive cases. Diabetes Care. 1996;19(6):638-41.

22. Kajetan M, Konkoly TM, Jermendy G. Experience with microbiologic studies of diabetic foot. Orv Hetil. 1995;136(40):2161-4.

23. Santoso M, Yuliana M, Mujono W. Kusdiantomo. Pattern of diabetic foot at Koja regional general hospital, Jakarta, from 1999 to 2004. Acta Med Indones. 2005;37(4):187-9.

24. Altrichter Loan C, Legout L, Assal M, Rohner P, Hoffmeyer P, Bernard L. Severe Streptococcus agalactiae infection of the diabetic foot: a deleterious role of Streptococcus agalactiae? Press Medicale. 2005;34(7):491-4.

25. Ng LSY, Lee LK, Yeow SCS, Thean YT. Anaerobic culture of diabetic foot infections: organisms and antimicrobial susceptibilities. Ann Acad Med Singap. 2008;37(11):936-9.

26. Bajuri MY, Abdul Razak KA. Chronic osteomyelitis of the femur with segmental bone defect: concepts and treatment. J Krishna Inst Med Sci Univ. 2017;6(2):127-30

27. Bajuri MY, Mohd RH. The physiological, biochemical and quality of life changes in chronic diabetic foot ulcer after hyperbaric oxygen therapy. Med Health. 2017;12(2):210-9.

28. Ge Y, MacDonald D, Hait H, Lipsky B, Zasloff M, Holroyd K. Microbiological profile of infected diabetic foot ulcers. Diabet Med. 2002;19(12):1032-4.

\section{Publisher's Note}

Springer Nature remains neutral with regard to jurisdictional claims in published maps and institutional affiliations.

Ready to submit your research? Choose BMC and benefit from:

- fast, convenient online submission

- thorough peer review by experienced researchers in your field

- rapid publication on acceptance

- support for research data, including large and complex data types

- gold Open Access which fosters wider collaboration and increased citations

- maximum visibility for your research: over $100 \mathrm{M}$ website views per year

At BMC, research is always in progress.

Learn more biomedcentral.com/submissions 